\title{
Dynamic Origin of Chirality Transfer between Chiral Surface and Achiral Ligand in $\mathrm{Au}_{38}$ Clusters
}

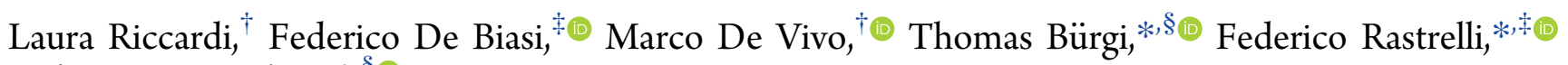 \\ and Giovanni Salassa*, ${ }^{\S}$ (ㅇ) \\ ${ }^{\dagger}$ Laboratory of Molecular Modeling \& Drug Discovery, Istituto Italiano di Tecnologia, Via Morego 30, 16163 Genova, Italy \\ ${ }^{\ddagger}$ Dipartimento di Scienze Chimiche, Università di Padova, Via Marzolo 1, 35131 Padova, Italy \\ ${ }^{\S}$ Department of Physical Chemistry, University of Geneva, 30 Quai Ernest-Ansermet, 1211 Geneva 4, Switzerland
}

Supporting Information

ABSTRACT: The transfer of chirality between nanomolecules is at the core of several applications in chiral technology such as sensing and catalysis. However, the origin of this phenomenon and how exactly nanoscale objects transfer chirality to molecules in their vicinity remain largely obscure. Here, we show that the transfer of chirality for the intrinsically chiral gold cluster $\mathrm{Au}_{38}(\mathrm{SR})_{24}$ is site dependent; that is, it differs depending on the ligand-binding sites. This is closely related to the dynamic nature of the ligands on the cluster surface. Using a combination of NMR techniques and molecular dynamics simulations, we could assign the four symmetryunique ligands on the cluster. The study reveals largely different conformational dynamics of the bound ligands, explaining the diverse diastereotopicities observed for the $\mathrm{CH}_{2}$ protons of the ligands. Although chirality is a structural property, our study reveals the importance of dynamics for the transfer of chirality.

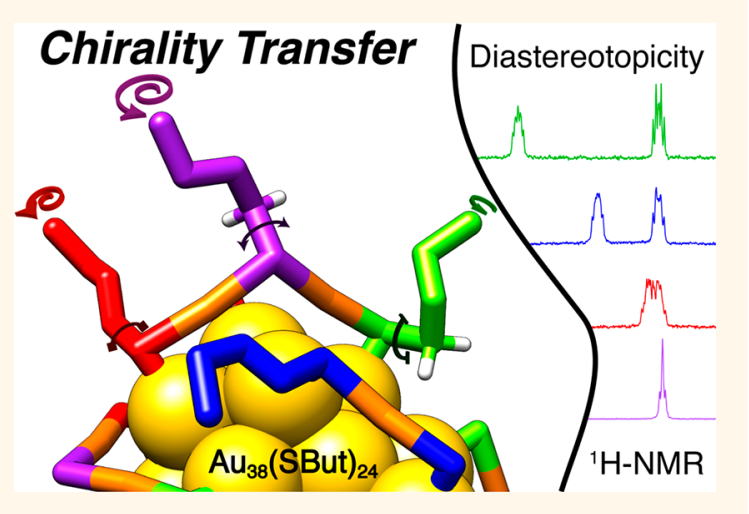

KEYWORDS: monolayer-protected clusters, chiral nanoclusters, chirality transfer, molecular dynamics simulations, A $u_{38}(S R)_{24}$ NMR, TOCSY

\begin{abstract}
$\mathrm{I}$ $\mathrm{n}$ the past decade, numerous examples of inorganic systems showing chiroptical properties at the nanoscale have been reported. ${ }^{1,2}$ Most of the efforts in creating inorganic chiral nanostructures are directed toward the combination of chiral (organic) molecules with metal nanoparticles. ${ }^{1}$ This involves the synthesis of nanoparticles characterized by a metal core stabilized by chiral organic or biomolecules. In these cases, the chirality originates from the organic ligands. However, the chiral ligand may alter the core structure, leading to the formation of "intrinsically chiral" objects. Very interesting examples of this chirality are represented by the small gold nanoparticles also called gold nanoclusters (AuNCs). As reported for the first time by Whetten and co-workers, small AuNCs protected by glutathione, a chiral cysteine-containing tripeptide, showed circular dichroism (CD) activity in electronic transitions that are localized mainly in the metal part. ${ }^{3}$ After publications of the first X-ray structure determinations of AuNCs, enormous advancements have been made in the comprehension of gold nanoclusters. $^{4-6}$ Some of the reported structures have been shown to possess an "intrinsic chirality" that arises from the
\end{abstract}

pattern created by the distribution of the "staple" motifs on the surface of the cluster. ${ }^{7}$ In this context, the term "staple" defines the bonding moiety of the thiolate ligands with the gold surface through the formation of linear monomeric RS-Au(I)-SR or dimeric $\mathrm{RS}-\mathrm{Au}(\mathrm{I})-\mathrm{SR}-\mathrm{Au}(\mathrm{I})-\mathrm{SR}$ complexes that cover the entire gold core.

The $\mathrm{Au}_{38}(2 \mathrm{PET})_{24}$ cluster (where 2PET is 2-phenylethylthiolate) is among the most studied examples of intrinsically chiral AuNCs. This cluster is characterized by a face-fused biicosahedral $\mathrm{Au}_{23}$ core protected by three monomeric and six dimeric staples (Figure 1). The staples are arranged in a chiral fashion: three dimeric staples on each pole of the cluster are disposed in a propeller-like configuration, which rotates clockwise or anticlockwise (but both in the same sense), depending on the enantiomer. Moreover, the monomeric staples at the equator of the cluster

Received: April 2, 2019

Accepted: June 6, 2019

Published: June 6, 2019 


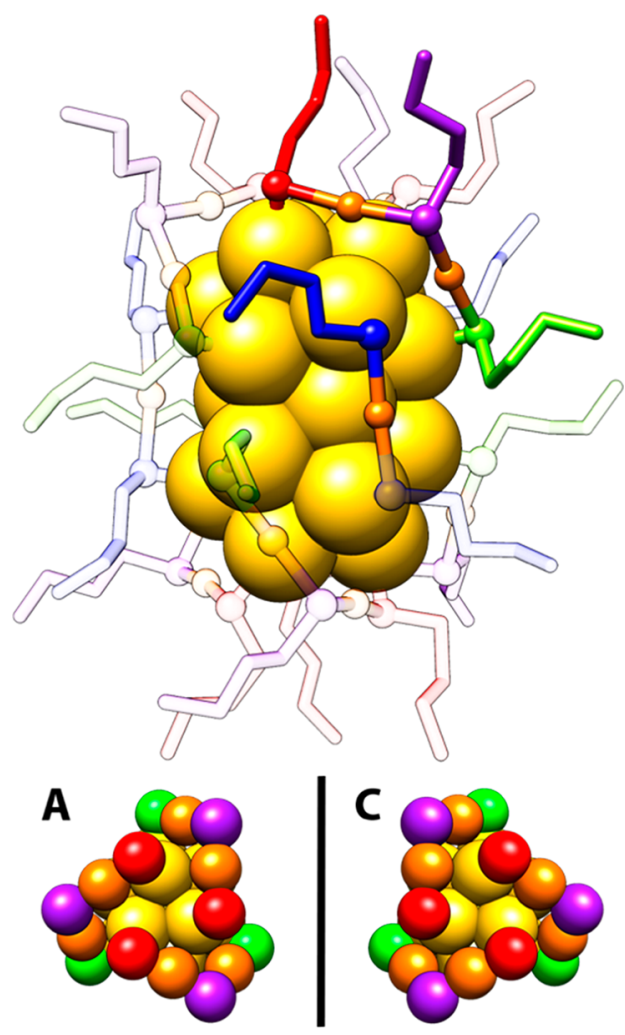

Figure 1. Structure of the $\mathrm{Au}_{38}(\mathrm{SBut})_{24}$ cluster. Top: The SBut ligands are colored accordingly with the four different symmetry environments. The $\mathrm{C}$ and $\mathrm{S}$ have the same color (20 But out of 24 are transparent for clarity), $\mathrm{Au}$ atoms in the core are in yellow, and the ones in the staples are in orange. Bottom: On the left the clockwise $\mathrm{C}$ enantiomer, on the right the anticlockwise $\mathrm{A}$ one (view from one pole). The structure is derived from the reported crystal structure of the $\mathrm{Au}_{38}(2 \mathrm{PET})_{24}$ cluster. $^{6}$

are slightly tilted with respect to the 3-fold axis, following the handedness of the long staples. We demonstrated previously that $\mathrm{C}$ and $\mathrm{A}$ (clockwise/anticlockwise) enantiomers of the $\mathrm{Au}_{38}(2 \mathrm{PET})_{24}$ cluster can be separated by chiral highperformance liquid chromatography (HPLC). ${ }^{8}$ The inversion between $\mathrm{C}$ and $\mathrm{A}$ has a surprisingly low energy barrier (ca. 28 $\mathrm{kcal} / \mathrm{mol})^{9}$ for a reaction including the breaking of $\mathrm{Au}-\mathrm{S}$ bonds. Indeed, a similar value for the activation barrier has been recently found by density functional theory (DFT) calculations for a mechanism that features a collective rotation of the gold core with no breaking of metal-sulfur bonds. ${ }^{10}$ The comparison between the $\mathrm{CD}$ spectra of $\mathrm{Au}_{38}(2 \mathrm{PET})_{24}$ with $\mathrm{Au}_{38}$ clusters protected with chiral glutathionate has shown a minor influence of the chiral ligand.

In a following study, we were able to observe a chirality transfer from the intrinsically chiral $\mathrm{Au}_{38}(2 \mathrm{PET})_{24}$ cluster to the bound achiral 2PET ligands using vibrational circular dichroism (VCD). ${ }^{11}$ In vibrational optical activity the term "chirality transfer" is employed when an achiral molecule shows optical activity due to its interaction with a chiral environment. ${ }^{12,13}$ The optical activity observed in this case arises from the "transiently" chiral conformations (gauche+ or gauche-) adopted by the $2 \mathrm{PET}$ (dihedral angle $\mathrm{S}-\alpha \mathrm{C}-\beta \mathrm{C}-$ $\mathrm{Ph}$ ) when bound to the cluster surface. These chiral conformations in the unbound 2PET are equally probable, resulting in no VCD activity. Nonetheless when 2PET is bound to one of the $\mathrm{Au}_{38}(2 \mathrm{PET})_{24}$ enantiomer ( $\mathrm{A}$ or $\mathrm{C}$ ), one of the chiral conformations of 2PET becomes more probable than the other. For this reason, the enantiopure $\mathrm{Au}_{38}(2 \mathrm{PET})_{24}$ shows a significant VCD signal. However, the VCD analysis provided a picture of the average behavior of $2 \mathrm{PET}$ bound to the surface. A precise understanding of the chiral conformations adopted (dynamically) by the 242 PET ligands is not yet achieved.

A few years before we reported the VCD investigation of $\mathrm{Au}_{38}(2 \mathrm{PET})_{24}$, Jin and co-workers observed that the intrinsically chiral $\mathrm{Au}_{38}$ surface affected the chemical shift $(\delta)$ of the 2PET's protons in NMR spectroscopy. ${ }^{14}$ Diastereotopic signals on the $\alpha \mathrm{C}$ and $\beta \mathrm{C}$ protons of 2PETs were observed. Diastereotopicity is usually described for protons belonging to the same $\mathrm{CH}_{2}$ group attached to (or in proximity to) a chiral stereocenter: since the two nuclei never experience the same chemical environment, they show different chemical shifts in the NMR spectra. For $\mathrm{Au}_{38}(2 \mathrm{PET})_{24}$, the "diastereotopicity" (i.e., the chemical shift difference $\Delta \delta$ ) induced on the $\alpha \mathrm{CH}_{2}$ protons is larger compared with an average $\mathrm{CH}_{2}$ in $\alpha$ at an asymmetric carbon. Moreover, the diastereotopic effect (in terms of $\Delta \delta$ ) is different for the four symmetry-unique ligand environments into which the 24 ligands of $\mathrm{Au}_{38}$ are divided (Figure 1). The authors, however, did not further investigate the origin of this diastereotopicity and also did not assign the NMR signals to the four different environments (that create four spin systems in the NMR spectra) with the Xray structure of $\mathrm{Au}_{38}(2 \mathrm{PET})_{24}{ }^{6}$

The diastereotopicity showed by $\mathrm{Au}_{38}$ is clearly related to the chirality transfer observed by VCD. For this reason, we decided to investigate in-depth the chiral properties of the representative $\mathrm{Au}_{38}(\mathrm{SBut})_{24}$ cluster (where SBut is butanethiolate). We employed mono- and multidimensional NMR spectroscopy together with molecular dynamics (MD) simulations to understand the origin of chirality transfer from the cluster to the ligand and to clarify how this phenomenon varies depending on the ligand-binding position. The combination of these two techniques has already provided useful information on the dynamic nature of the thiolate monolayer in AuNCs, ${ }^{15}$ like in the case of $\mathrm{Au}_{102}(\mathrm{pMBA})_{44}{ }^{16,17}$

Recently Maran and co-workers reported an NMR analysis of propanethiolate- and butanethiolate-protected $\mathrm{Au}_{38}{ }^{18}$ The authors were able to assign two of the observed signals out of four symmetry-unique ligands by comparing solid-state structural data with NMR in solution. The NMR/MD approach employed allowed us not only to confirm the assignation hypothesized by Maran and co-workers but also to complete it with respect to the four symmetry-unique ligands of the $\mathrm{Au}_{38}$.

\section{RESULTS AND DISCUSSION}

Preparation and General Considerations. We have prepared $\mathrm{Au}_{38}(\mathrm{SBut})_{24}$ clusters (where SBut is butanethiolate) as described in the Methods section. Full characterization of the cluster is reported in the Supporting Information (Figure $\mathrm{S} 1$ ), and it is in agreement with previous reports. ${ }^{8,14}$ Butanethiol was selected as the protecting ligand due to its less hindered nature compared with $2 \mathrm{PET}$. This creates a more flexible ligand monolayer around the cluster that is easier to study by NMR and MD. The experiments were carried out in $n$-hexane- $d_{14}$ for two reasons. First, the linear structure of the solvent enables it to interdigitate with the butanethiolate ligands on the cluster, allowing the latter to move with less constraints. The second reason is the good stability observed 

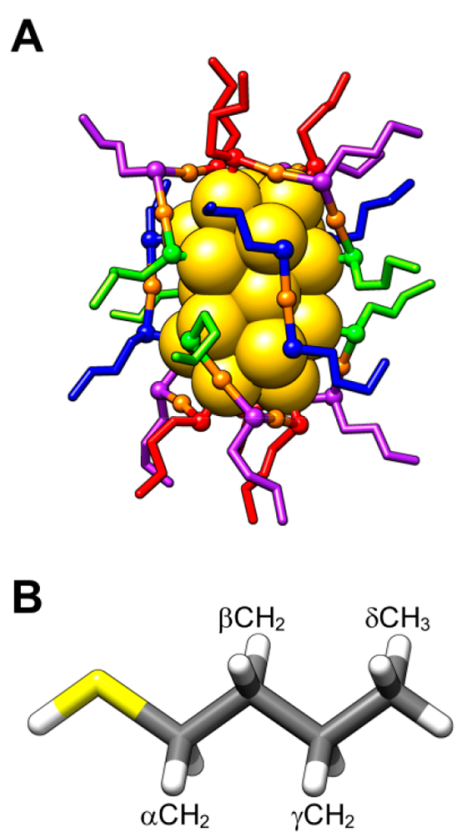

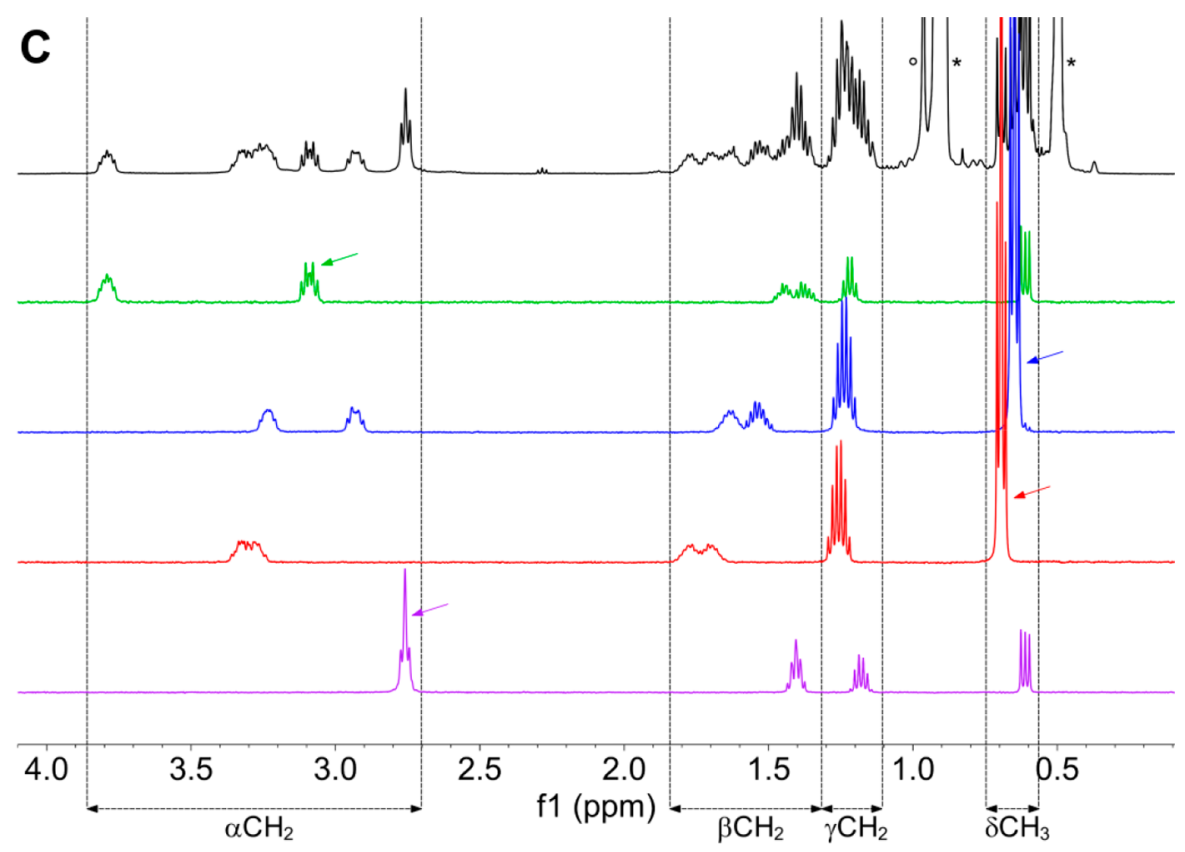

Figure 2. CSSF-TOCSY spectra of the $\mathrm{Au}_{38}(2 \mathrm{PET})_{24}$ clusters (A) $0.83 \mathrm{mM}$ in $n$-hexane- $d_{24}$ at $45^{\circ} \mathrm{C}$. The arrows denote the chemical shifts in the four different groups of butyl thiol chains (B) that have been selectively filtered. These are the starting points for the TOCSY magnetization propagation, which was achieved through a $70 \mathrm{~ms}$ DIPSI2 mixing scheme.

for $\mathrm{Au}_{38}(\mathrm{SBut})_{24}$ in this solvent. The different NMR tubes prepared with $\mathrm{Au}_{38}(\mathrm{SBut})_{24}$ in $n$-hexane- $d_{14}$ showed no sign of decomposition over 6 months of testing. On the other hand, a potential solvent candidate such as dichloromethane- $d_{2}$ induced decomposition of the cluster, which formed a peculiar golden patina on the tube's glass walls, in only a few hours.

The NMR analysis of $\mathrm{Au}_{38}(2 \mathrm{PET})_{24}$ reported by Jin and coworkers ${ }^{14}$ showed that the 24 ligands can be grouped into four different sets of magnetically equivalent ligands. These sets of ligands correspond to the four symmetry-unique ligand environments originated from the symmetry of the cluster core. By inspecting the three-dimensional structure of the cluster (Figure 1), a fusion plane can be found at the equator of $\mathrm{Au}_{38}$, passing in the middle of the monomeric staples. The ligands above and below the fusion plane are symmetry equivalent. The $\mathrm{C}_{3}$ rotation axis, passing through the poles, makes the three dimeric (SR-Au-SR-Au-SR) staples on each hemisphere equivalent. Thus, the three -SR ligands in each dimeric staple correspond to symmetry-unique ligand environments. The fourth symmetry-unique ligand environment derives from the monomeric staples. The same analysis is valid for the $\mathrm{Au}_{38}(\mathrm{SBut})_{24}$ cluster. For the sake of clarity we colored the four symmetry-unique ligand environments differently: the purple chain group binds to the central sulfur atom of the dimeric staple, while the red and green chain groups bind the sides of this staple but at the pole and at the equator of the cluster, respectively. Finally, the blue chain group binds to the monomeric staple, which is centered at the equator of the cluster, where the curvature is minimal (Figure $1)$.

NMR Experiments and Chemical Shift Assignment of $\mathrm{Au}_{38}$ (SBut) 24 . CSSF TOCSY. Total correlation spectroscopy (TOCSY) represents a powerful method to assign the NMR signals of protons belonging to a specific spin system. While this experiment is usually employed to generate two-dimensional maps (see, e.g., ref 17 for a typical application to gold nanoclusters), inclusion of selective excitation RF pulses into the pulse sequence leads to a one-dimensional TOCSY spectrum featuring a much higher resolution with respect to its two-dimensional counterpart. In our case, 1D TOCSY complemented with chemical-shift selective filters (CSSF, see Methods) proved to be an ideal tool to unravel the ${ }^{1} \mathrm{H}$ NMR spectrum of $\mathrm{Au}_{38}(\mathrm{SBut})_{24}$, since each (symmetry-equivalent) butylthiolate chain group represents an isolated spin system whose subspectrum can be conveniently extracted from the total spectrum. To this aim, the most straightforward starting point for the propagation of the magnetization is indeed the methyl resonance; careful inspection of the ${ }^{1} \mathrm{H}$ NMR spectrum however reveals that two methyl signals are perfectly overlapped. This stumbling block was overcome by selecting other well-resolved signals, emphasized in Figure 2. The 1D TOCSY spectra highlighted in a clear fashion the different diastereotopicity displayed by the $\alpha \mathrm{CH}_{2}$ and $\beta \mathrm{CH}_{2}$ in three out of the four butyl chain groups (spin systems). In particular, the green chain group showed the highest diastereotopicity, whereas the purple one displayed a TOCSY spectrum very close to the free butanethiol.

${ }^{13} \mathrm{C}-T_{1}$. In order to highlight possible differences among the dynamics of the different chain groups, ${ }^{13} \mathrm{C}$ relaxometry experiments were performed and the results compared with predictions made by $\mathrm{MD}$ simulations. The longitudinal relaxation time $T_{1}$ of each ${ }^{13} \mathrm{C}$ nucleus depends essentially on the fluctuations of the dipole-dipole interactions between the carbon and the directly attached protons (neglecting chemical shift anisotropy mechanisms), thus returning an insight into the local dynamics of the chain groups. Moreover, the same quantity can also be estimated from MD simulations (e.g., via the Lipari-Szabo approach, vide infra). The comparison between calculated and experimental values (Table 1) was in good agreement and allowed confirming the assignments of the purple and the green chain groups, which exhibit the longest and the shortest values of $T_{1}$, 
Table 1. Calculated and Experimental Values of ${ }^{13} \mathrm{C} T_{1}$ (in s)

\begin{tabular}{lcccc} 
& $\alpha C_{\text {exp }}$ & $\alpha C_{\text {calc }}$ & $\beta C_{\text {exp }}$ & $\beta C_{\text {calc }}$ \\
purple & $0.365 \pm 0.026$ & 0.519 & $0.887 \pm 0.045$ & 0.754 \\
blue & $0.333 \pm 0.023^{a}$ & 0.346 & $0.591 \pm 0.031$ & 0.438 \\
red & $0.333 \pm 0.023^{a}$ & 0.322 & $0.565 \pm 0.046$ & 0.416 \\
green & $0.239 \pm 0.022$ & 0.267 & $0.512 \pm 0.025$ & 0.324 \\
\multicolumn{2}{l}{${ }^{a}$ Same $T_{1}$ value. } & & &
\end{tabular}

respectively. In this analysis, the blue and red chains remain elusive, since they share the same $T_{1}$ within experimental error.

$1 D$ NOESY. In order to search for proximities among the different chain groups, selective NOESY spectroscopy was employed. In this case, the triplet belonging to the $\alpha \mathrm{CH}_{2}$ purple chain group was a rather straightforward choice as the source of magnetization to be transferred via NOE. The $1 \mathrm{D}-$ NOESY spectrum obtained by selective inversion of the aforementioned triplet displays resonances of the $\beta \mathrm{CH}_{2}, \gamma \mathrm{CH}_{2}$, and $\delta \mathrm{CH}_{3}$ belonging to the same chain, as can be verified from Figure 3 . The opposed polarity with respect to the inverted

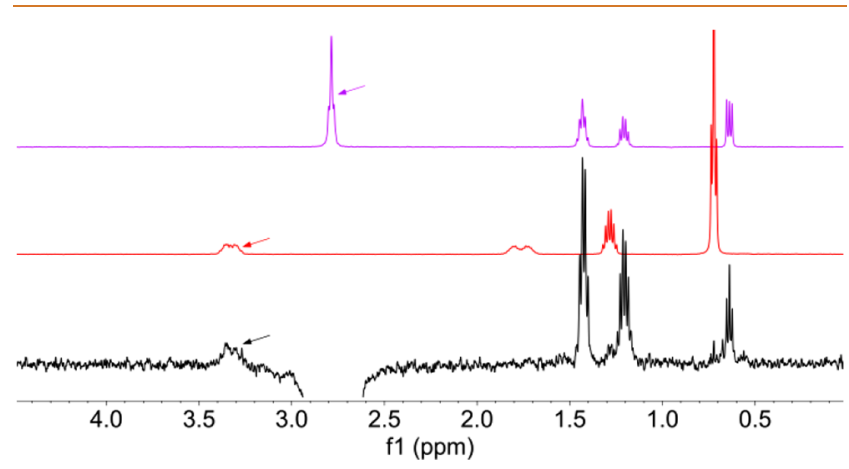

Figure 3. 1D-NOESY spectrum (black) of $\mathrm{Au}_{38}(2 \mathrm{PET})_{24}$ clusters upon selective inversion of the signal at $2.75 \mathrm{ppm}$ (mixing time = $0.60 \mathrm{~s}$ ). The purple (top) trace recalls the CSSF-TOCSY of the purple chain in Figure 2. The weak signal at $3.4 \mathrm{ppm}$ has a unique correspondence in the CSSF-TOCSY spectrum of the (supposedly) red chain. See text for details.

signal indicates a positive NOE regime and unveils a relatively short (effective) correlation time, a situation rather different from $2 \mathrm{~nm}$ core nanoparticles, among other examples.

Interestingly, the same 1D-NOESY spectrum displays a weak signal at about $3.3 \mathrm{ppm}$ belonging to the $\alpha \mathrm{CH}_{2}$ protons of another chain group, whose subspectrum could be attributed by comparison with CSSF-TOCSY traces (Figure 2). On the basis of the averaged distances between the $\alpha \mathrm{CH}_{2}$ protons, this subspectrum is tentatively attributed to the red chain group, which displays the closest contacts with the purple chain group. The same NOE contact was observed in the recent report by Maran and co-workers. ${ }^{18}$

Conformation Analysis by MD. Dynamics. The time scale of internal molecular motions of small nanoparticle coating ligands is accessible by means of extensive MD simulations in explicit solvent. ${ }^{19}$ For each $\mathrm{CH}$ bond vector, the autocorrelation of the second-order Legendre polynomial was calculated from the MD trajectories after removing the overall tumbling and translational motions (Figure S7). In this way, only internal degrees of freedom are taken into account. For all the different butyl thiol chain groups (i.e., purple, red, green, blue), the autocorrelation decreases faster while moving along the coating thiol carbons, from $\alpha \mathrm{CH}_{2}$ to $\delta \mathrm{CH}_{3}$, reflecting that the inner methylenes are always more constrained than the outer ones (Figure S8).

The time correlation functions of the $\mathrm{CH}$ vectors were fitted by a biexponential curve. ${ }^{20}$ The results of the fitting were used to calculate the spin-lattice relaxation time $T_{1}$ values for ${ }^{13} \mathrm{C}$ relaxation as a function of the external field, via the LipariSzabo approach (see the Supporting Information). ${ }^{21}$ Comparing the computed $T_{1}$ relaxation values of the different butylthiolate chain groups, the purple profile clearly emerges at higher $T_{1}$ values, for all carbons. In contrast, the green profile has a slightly lower relaxivity compared to the two remaining butylthiolate chain groups.

Distances. We calculated the NMR averaged distances between the $\alpha \mathrm{CH}_{2}$ protons of all the butylthiolate chains. The interproton distances were computed as $r_{p}=\left\langle r^{-p}\right\rangle^{-1 / p}$, where $p$ is 3 or 6 , over the structures of the whole simulation time (Figure S9). The $r_{3}$ or $r_{6}$ averaging of the internuclear distances biases the ensemble average toward the left tail of the distance distribution, allowing a comparison with NMR distances. The $r_{3}$ averaging is mainly used for small molecules, where the internal fluctuations are slower than the tumbling time, while $r_{6}$ is used for bigger molecules, in which the internal fluctuations are faster than the tumbling time. The $\alpha \mathrm{CH}_{2}$ protons of the purple group form contacts, within $4 \AA$, only with the $\alpha \mathrm{CH}_{2}$ protons of the red group. Increasing the distance threshold to 5 $\AA$, it is possible to capture some long-range contacts with the blue chain group. This result is consistent in both $r_{3}$ and $r_{6}$ averaging method.

Dihedral Angles. Dihedral angle fluctuations are a source of internal motion, and their analysis provides insights into the dynamics of the coating ligands. We calculated the rotamer population of the dihedral angles of the coating ligands from our $\mathrm{MD}$ simulations. The $\mathrm{Au}-\mathrm{S}-\alpha \mathrm{C}-\beta \mathrm{C}$ dihedral angle has three main conformational minima for each flip of the sulfur atom, from one side of the staple to the other (Scheme 1). The rotamer population analysis reveals a conformational heterogeneity of the different butylthiolate chain groups (vide infra).

The next dihedral angle $\mathrm{S}-\alpha \mathrm{C}-\beta \mathrm{C}-\gamma \mathrm{C}$ was also investigated. In all chain groups, the anti conformation is the predominant one, being populated at least $50 \%$ of the simulation time (Figure S11). The remaining population equally distributes between the two gauche conformations.

Solvation. The solvent accessible surface (s.a.s.) area was calculated during the MD simulations, for each butylthiolate chain and chain group. The green chain group, which is located at the equatorial side of the dimeric staples, had an s.a.s. area of $4.4 \pm 0.3 \mathrm{~nm}^{2}$. The blue and red chain groups have a better solvation ( $4.9 \pm 0.3$ and $5.0 \pm 0.3 \mathrm{~nm}^{2}$, respectively). Still, their distribution of the accessible surface area overlaps the green one. The purple chain group, however, has the highest solvation, $7.0 \pm 0.3 \mathrm{~nm}^{2}$, and this is due mainly to an increased solvent accessibility of the inner methylenes (see Figure S12).

Diastereotopicity and Ligand Dynamics. Dihedral Conformations of $\mathrm{Au}-\mathrm{S}-\mathrm{C} \alpha-\mathrm{C}_{\beta}$ and Population Analysis. We first note from the experimental CSSF-TOCSY spectra that the diastereotopicity extends also to the beta protons, so that each chain group (other than the purple) apparently gives rise to an $A B-M L-X_{2}-Y_{3}$ spin system. At a first analysis, the $\mathrm{S}-\mathrm{CH}_{2}-$ protons are located in alpha with respect to a chiral center represented by the sulfur atom. In this perspective, such protons in the green, red, and blue chain groups are formally 
Scheme 1. Minimum energy gauche and syn conformations of the different chains (same color code as before) and their relative populations, as obtained from $\mathrm{MD}$ simulations.

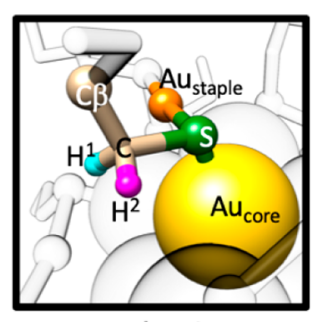

gauche 1

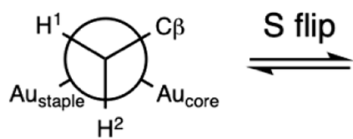

$14 \% 9 \% 7 \% 6 \%$<smiles>C=C=C</smiles>

gauche 2

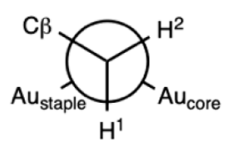

$13 \% 13 \% 8 \% 40 \%$

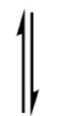

syn

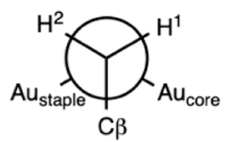

$24 \% 33 \% 22 \% 51 \%$

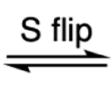

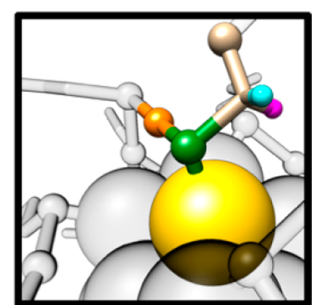

gauche 1

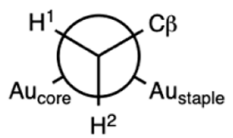

$13 \% \quad 8 \% 10 \% 1 \%$

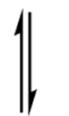

gauche 2

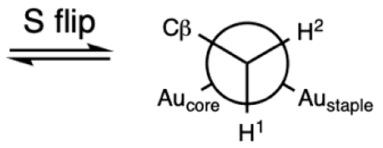

$11 \% 9 \% 31 \% 1 \%$

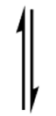

syn

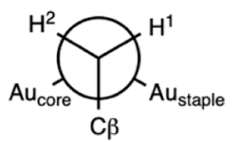

$24 \% 28 \% 22 \% \quad 2 \%$ diastereotopic. The case of the $\alpha \mathrm{CH}_{2}$ protons in the purple chain group stands apart, since the sulfur here is grafted in the central position of the dimeric staple $\left(\mathrm{Au}_{\text {staple }}-\mathrm{S}-\mathrm{Au}_{\text {staple }}\right)$, which eliminates part of the asymmetry. Indeed, the experimental spectrum of the purple chain group highlights an $\mathrm{A}_{2} \mathrm{M}_{2} \mathrm{X}_{2} \mathrm{Y}_{3}$ spin system with no apparent diastereotopicity.

The rationalization of the diastereotopic effect is however complicated by the fact that the flip of the $\mathrm{S}-\alpha \mathrm{C}$ bond about the $\mathrm{S}$ center has a low activation energy (for $\mathrm{Au}_{102}$ and $\mathrm{Au}_{25}$ clusters, DFT calculations estimated a $0.3 / 0.6 \mathrm{eV}$ barrier for the flip of the central $S$ in dimeric staples), ${ }^{22}$ so that the relative configuration of the $S$ center could invert fast on the NMR time scale, in much the same way as amines do. The effect of the $S$ flip is to endow a (double) two-site exchange: indeed, in the fast exchange regime, the observed resonances lie at an average position weighted over the populations of the two rotamers (apart from the chemical shifts). Only in the case of equal populations do the two $\mathrm{CH}_{2}$ protons give rise to one signal. Spectra acquired at different temperatures (from 10 to $45{ }^{\circ} \mathrm{C}$, Figure S2) do not display a significant perturbation of the chemical shifts, but only a progressive sharpening of the lines with increasing temperatures, indicating that the system is already in the fast exchange regime over the different rotamers.
In the Newman projections, the $S$ inversion can be conveniently visualized by exchanging the $\mathrm{Au}_{\text {staple }}$ and $\mathrm{Au}_{\text {core }}$ atoms. The analysis of the relative populations (Scheme 1) points out that the green chain group exhibits a very uneven distribution of populations, with the two most probable rotamers confined to one conformation of the S-flip. Compared with the green chain group, the blue and red chain groups have a less unbalanced rotamer distribution, in agreement with the lower observed diastereotopicity. For the purple chain group, which shows no diastereotopic effect on the methylene chemical shifts, the syn conformation is the predominant one, and the two gauches are equally populated. Moreover, the population of the states is symmetric with respect to the S-flip.

In our case, the unbalance in the rotamer population follows the internal dynamics of the different chain groups, from the least flexible and least balanced (the green chain group) to the most flexible and most balanced (purple). That is, the internal molecular motions and the equilibrium populations of every ligand are highly interconnected. In this context the case of $\mathrm{Au}_{38}(2 \mathrm{PET})_{24}{ }^{14}$ where a bulky phenyl group replaces the $\gamma \mathrm{CH}_{2}$, provides a further example of the effects that we ascribe to the conformational dynamics. Notably, the presence of a hindered moiety is expected to reduce the conformational space accessible to each ligand, enhancing the unbalance between rotamer populations and, consequently, the diastereotopic effect. Indeed, in this system, diastereotopicity is observed for all four symmetry-unique ligands. ${ }^{14}$

Dihedral Conformations of $S-\alpha C-\beta C-\gamma C$ and Population Analysis. The distribution of the $\mathrm{S}-\alpha \mathrm{C}-\beta \mathrm{C}-\gamma \mathrm{C}$ dihedral angle states in the purple and red chain groups (Figure S11) resembles that of free $n$-butane. Previous simulations as well as electron diffraction studies reported an anti population of $67.8 \%{ }^{23}$ and $60 \pm 15 \%,{ }^{24}$ respectively, for $n$-butane. For the case of green and blue chain groups, the two gauche conformations become more probable, indicating that the surrounding environment (i.e., the asymmetric disposition of ligands on the surface) drive this dihedral angle toward the less stable conformations. The different populations observed for the red and the blue chain groups might be explained by their position on the cluster surface. The red chain group binds the gold core at the pole, where the curvature is maximum, meanwhile the blue chain group stands on the equator, where the curvature is minimal. The difference in diastereotopicity observed between red and blue chain groups might be partially rationalized by different mobility calculated on the second dihedral angle.

We suggest that the unbalance in the populations of each chain group can be seen as the result of the formation of "super-diastereoisomers" of the kind $\mathrm{C}-\mathrm{R}$ and $\mathrm{C}-\mathrm{S}$ (or $\mathrm{A}-\mathrm{R}$ and $A-S$ ), where $R$ and $S$ define the local asymmetry of the sulfur atom, while $\mathrm{C}$ and A define the chirality of the cluster (Figure 1). In summary, the emerging picture is one wherein the "diastereotopic effect" should be proportional (among other factors such as the intrinsic chemical shifts) to the unbalance in the equilibrium populations of the (fast) interchanging super-diastereoisomers.

Differences between $\mathrm{Au}_{25}(\mathrm{SBut})_{18}$ and $\mathrm{Au}_{38}(\mathrm{SBut})_{24}$. The comparison reported by Jin and co-workers ${ }^{14}$ between $\mathrm{Au}_{25}(2 \mathrm{PET})_{18}$ and $\mathrm{Au}_{38}(2 \mathrm{PET})_{24}$ clearly shows the effect of the $\mathrm{Au}_{38}$ intrinsic chirality on the ${ }^{1} \mathrm{H}$ NMR. In the case of the achiral $\mathrm{Au}_{25}(2 \mathrm{PET})_{18}$ no diastereotopic effect is observed on the $2 \mathrm{PET}$ protons, and the same behavior is observed in the 
reported ${ }^{1} \mathrm{H}$ NMR of $\mathrm{Au}_{25}(\mathrm{SBut})_{18} \cdot{ }^{25}$ This comparison demonstrates how the chiral distribution of the staples creates the asymmetric environment that affects the populations equilibrium of every ligand, explaining the origin of diastereotopicity. Analyzing the staple distribution of $\mathrm{Au}_{25}$ (Figure S13), a highly symmetric environment is created around every ligand. Therefore, the dynamics of every ligand are not affected in this way to induce a diastereotopic effect.

\section{CONCLUSION}

In our work, the interpretation of the complex ${ }^{1} \mathrm{H}$ NMR spectra of the $\mathrm{Au}_{38}(\mathrm{SBut})_{24}$ cluster was achieved by a combination of high-resolution 1D NMR methods and extensive MD simulations. First, by comparing the experimental and the MD-calculated $T_{1}$ values for ${ }^{13} \mathrm{C}$ (Table 1 and Figure S8), it was possible to assign the purple and green chain groups. The red and blue chain groups could not be discriminated yet due to similar relaxation profiles. From an HSQC experiment (Figures S3 and S4) we then correlated the ${ }^{13} \mathrm{C}$ signals of green and purple chain groups with their respective proton signals. In order to complete the assignment, we looked for possible interligand NOE contacts between the $\alpha \mathrm{CH}_{2}$ protons of the different chains (Figure 3). 1D NOESY experiment shows only one interaction between the purple and one of the unknown chain groups (red or blue). From the map of averaged distances between the $\alpha \mathrm{CH}_{2}$ protons calculated with $\mathrm{MD}$, the most likely interaction observed for the purple chain group was with the red one (within $4 \AA$ ). With this piece of information, we were able to assign all four spin systems (see CSSF-TOCSY, Figure 2) to their corresponding four groups of the butyl thiolate chain.

The chirality transfer between $\mathrm{Au}_{38}$ and the bound ligands was observed by VCD in solution. ${ }^{11}$ The achiral ligand (in that case 2PET) became VCD active upon binding with the intrinsically chiral $\mathrm{Au}_{38}$. The optical activity observed with this technique arises from the unbalanced abundance of the two possible forms of gauche conformations (vide supra). Similarly, NMR techniques were able to observe the chirality transfer for the $\mathrm{Au}_{38}$ system. The analysis of the diastereotopicity, as presented here, clearly revealed that the chirality transfer is distinctly different for the different binding sites of $\mathrm{Au}_{38}$. From the examination of the MD simulations we showed how the mobility of each type of ligand is affected differently by the chiral disposition of the staples in $\mathrm{Au}_{38}$ : from the least affected ligand (the purple chain group) to the most affected one (green). The information obtained on the $\mathrm{Au}_{38}$ thiolate monolayer allowed us to understand the dynamic aspect of the chirality transfer phenomenon, influencing greatly the future applications of these systems.

The possibility to foresee how every ligand is affected by the $\mathrm{Au}_{38}$ surface provides the necessary tool to optimize the ligand structure in such a way as to obtain the highest possible chirality transfer. This would allow understanding and controlling the asymmetric interactions that the $\mathrm{Au}_{38}$ monolayer would establish with the external environment (e.g., molecules, chiral surfaces, etc.). These asymmetric interactions could be employed for the creation of a type of chiral recognition based on $\mathrm{Au}_{38}(\mathrm{SR})_{24}$ nanosystems, fostering potential applications in sensing and catalysis.

\section{MATERIALS AND METHODS}

Sample Preparation. $\mathrm{Au}_{38}(\mathrm{SBut})_{24}$ clusters were prepared according to previously reported protocols. ${ }^{26,27}$ All the samples were purified through size-exclusion chromatography (SEC) in order to achieve purely monodispersed compounds. ${ }^{28}$ In summary, tetrachloroauric acid and L-glutathione were dissolved in acetone and reduced by sodium borohydride $\left(\mathrm{NaBH}_{4}\right)$. The resulting clusters were then dissolved in water, and a mixture of ethanol, toluene, and thiol (2PET or butanethiol) was added. The solution was heated at 80 ${ }^{\circ} \mathrm{C}$ for $30 \mathrm{~h}$ to size-focus the mixture of $\mathrm{Au}_{n}(2 \mathrm{PET})_{m}(n: 25-144, m=$ 18-60) toward $\mathrm{Au}_{38}(\mathrm{SR})_{24}$ clusters. Excess thiol was removed by extensive methanol washing, and the crude clusters were size-selected by SEC. For more details see the Supporting Information. The pure $\mathrm{Au}_{38}(\mathrm{SBut})_{24}$ clusters were characterized by MALDI mass spectrometry and UV-vis spectroscopy (Figure S1), which showed the characteristic mass and optical transitions of the cluster.

NMR Spectroscopy. All NMR experiments except inversion recovery for the measurement of the ${ }^{13} \mathrm{C}$ relaxation were performed on a Bruker Avance III spectrometer operating at $500.13 \mathrm{MHz}$ proton Larmor frequency and equipped with a $5 \mathrm{~mm} z$-gradient broadband inverse (BBI) probe. Inversion recovery was performed on a Bruker AVANCE DRX 500 equipped with a $5 \mathrm{~mm} z$-gradient TCI ${ }^{1} \mathrm{H}-{ }^{13} \mathrm{C} /{ }^{15} \mathrm{~N} /{ }^{2} \mathrm{H}$ cryoprobe. All NMR samples were prepared in perdeuterated $n$-hexane and were equilibrated at $318.1 \mathrm{~K}\left(45{ }^{\circ} \mathrm{C}\right)$ inside the spectrometer before signal acquisition.

One-dimensional TOCSY can be obtained by means of selective RF pulses, leading to a much higher spectral resolution with respect to the two-dimensional counterpart. In many cases, however, the occurrence of overlapping signals often requires a selectivity beyond the reach of standard shaped (RF-modulated) pulses. CSSFs provide an effective solution to this problem. Different from shaped pulses, the excitation profile of CSSFs results from the constructive addition of on-resonance signals, while off-resonance magnetization components are eliminated by destructive interference. In 2004, Robinson et al. ${ }^{29}$ substantially improved the performances of CSSFs by complementing the original idea of Hall and Norwood with pulsed field gradients (PFGs) ${ }^{30}$ Further refinements of the gradient-enhanced CSSF have been subsequently proposed by Duncan et al. ${ }^{31}$ Since only in-phase magnetization emerges from the filter, a CSSF can be conveniently followed by a mixing scheme, leading to highly selective $1 \mathrm{D}$-analogues of $2 \mathrm{D}$ experiments such as NOESY or TOCSY. In the latter case, the cluster of RF pulses that drives the isotropic mixing also introduces a slight heating of the sample, which may ultimately displace the resonances by a few Hz. Due to the high selectivity of the CSSF, such a slight offset can be detrimental for the experimental output and must be carefully accounted for.

For the CSSF-TOCSY experiments the acquisition parameters are 48 scans $\times 14$ increments; $6000 \mathrm{~Hz}$ spectral width on $8 \mathrm{k}$ points; 1.36 $\mathrm{s}$ acquisition time; $2 \mathrm{~s}$ relaxation delay; $70 \mathrm{~ms}$ DIPSI 2 mixing scheme with zero-quantum coherence suppression. With these parameters, the duration of the CSSF-TOCSY experiment was about $40 \mathrm{~min}$. For the selective NOE experiment the acquisition parameters are 2800 scans; $6000 \mathrm{~Hz}$ spectral width on 8k points; $1.20 \mathrm{~s}$ acquisition time; $3 \mathrm{~s}$ relaxation delay; $0.7 \mathrm{~s}$ mixing time. The total duration of the experiment was about $3 \mathrm{~h}$ and $50 \mathrm{~min}$. For the ${ }^{13} \mathrm{C}$ inversion recovery the parameters are 1536 scans $\times 9$ sampled delays (maximum delay of $3.5 \mathrm{~s}) ; 8800 \mathrm{~Hz}$ spectral width on $8 \mathrm{k}$ points; $0.93 \mathrm{~s}$ acquisition time; 2 $\mathrm{s}$ relaxation delay. With these parameters the total duration of the experiment was about $16 \mathrm{~h}$ and $20 \mathrm{~min}$.

Computational Methods. $\mathrm{Au}_{38}$ (SBut $_{24}$ Model. The computational model of $\mathrm{Au}_{38}(\mathrm{SBut})_{24}$ was based on the $\mathrm{Au}_{38}(2 \mathrm{PET})_{24}$ structure, ${ }^{6}$ in which $-\mathrm{Ph}$ was substituted with $-\mathrm{CH}_{2} \mathrm{CH}_{3}$. The coating groups and the $n$-hexane solvent were parametrized according to the Lipid14-ff, ${ }^{32}$ while the atomic charges were derived by the RESP $^{33}$ fitting procedure, calculated via RedServer. ${ }^{34}$ Additional bond and angle parameters were added to the force field to keep the gold/ monolayer interface stable. ${ }^{22}$ The $\mathrm{Au}_{38}(\mathrm{SBut})_{24}$ cluster was immersed in a dodecahedral simulation box, which was used to ensure a minimum distance between the $\mathrm{Au}_{38}(\mathrm{SBut})_{24}$ and the box edges of at least $2 \mathrm{~nm}$, and filled up with the pre-equilibrated $n$-hexane molecules. The system was slowly equilibrated in several steps to reach the final temperature; the system was slowly thermalized in different steps and then pressurized. Production runs were carried out in the NPT $(p=1$ 
bar, $T=318 \mathrm{~K}$ ) statistical ensemble, using a time step set of $2 \mathrm{fs}$. Periodic boundary conditions were applied to the systems in all directions. A total of $650 \mathrm{~ns}$ long MD simulations were performed using GROMACS-4.6. ${ }^{35}$ Coordinates of the systems were collected every 2 ps. Additional details about the MD protocol and the computational analysis can be found in the Supporting Information.

\section{ASSOCIATED CONTENT}

\section{S Supporting Information}

The Supporting Information is available free of charge on the ACS Publications website at DOI: 10.1021/acsnano.9b02552.

Further details on the characterization of $\mathrm{Au}_{38}(\mathrm{SBut})_{24}$, NMR spectroscopy, and molecular dynamics investigation (PDF)

\section{AUTHOR INFORMATION}

\section{Corresponding Authors}

*E-mail: thomas.buergi@unige.ch.

*E-mail: federico.rastrelli@unipd.it.

*E-mail: Giovanni.Salassa@unige.ch.

\section{ORCID}

Federico De Biasi: 0000-0001-6548-8383

Marco De Vivo: 0000-0003-4022-5661

Thomas Bürgi: 0000-0003-0906-082X

Federico Rastrelli: 0000-0002-2369-2228

Giovanni Salassa: 0000-0002-2396-3884

\section{Author Contributions}

G.S. prepared the clusters. F.D. and F.R. performed the NMR measurement. L.R. performed the MD calculations. G.S. and T.B. designed the concept of the work. All authors contributed to the writing of the manuscript. The project was supervised by G.S. and F.R.

\section{Notes}

The authors declare no competing financial interest.

\section{ACKNOWLEDGMENTS}

We gratefully acknowledge financial support from the University of Geneva, and the Swiss National Science Foundation (grant number 200020 172511) is kindly acknowledged. G.S. thanks EU Horizon 2020 Marie Skłodowska-Curie Action IF GOLDENSENS 747209 for financial support. Access to the Centro di Risonanze Magnetiche (CERM) infrastructure (Florence) provided by Mario Piccioli and technical support from Fabio Calogiuri are gratefully acknowledged. We also thank Prof. Fabrizio Mancin for the numerous and helpful discussions.

\section{REFERENCES}

(1) Ma, W.; Xu, L.; De Moura, A. F.; Wu, X.; Kuang, H.; Xu, C.; Kotov, N. A. Chiral Inorganic Nanostructures. Chem. Rev. 2017, 117, 8041-8093.

(2) Kumar, J.; Thomas, K. G.; Liz-Marzán, L. M. Nanoscale Chirality in Metal and Semiconductor Nanoparticles. Chem. Commun. 2016, 52, 12555-12569.

(3) Schaaff, T. G.; Whetten, R. L. Giant Gold-Glutathione Cluster Compounds: Intense Optical Activity in Metal-Based Transitions. J. Phys. Chem. B 2000, 104, 2630-2641.

(4) Jadzinsky, P. D.; Calero, G.; Ackerson, C. J.; Bushnell, D. A.; Kornberg, R. D. Structure of a Thiol Monolayer-Protected Gold Nanoparticle at 1.1 Å Resolution. Science 2007, 318, 430-433.

(5) Heaven, M. W.; Dass, A.; White, P. S.; Holt, K. M.; Murray, R. W. Crystal Structure of the Gold Nanoparticle $\left[\mathrm{N}\left(\mathrm{C}_{8} \mathrm{H}_{17}\right)_{4}\right]$ $\left[\mathrm{Au}_{25}\left(\mathrm{SCH}_{2} \mathrm{CH}_{2} \mathrm{Ph}\right)_{18}\right]$. J. Am. Chem. Soc. 2008, 25, 3754-3755.
(6) Qian, H.; Eckenhoff, W. T.; Zhu, Y.; Pintauer, T.; Jin, R. Total Structure Determination of Thiolate-Protected Au38 Nanoparticles. J. Am. Chem. Soc. 2010, 25, 8280-8281.

(7) Knoppe, S.; Bürgi, T. Chirality in Thiolate-Protected Gold Clusters. Acc. Chem. Res. 2014, 47, 1318-1326.

(8) Dolamic, I.; Knoppe, S.; Dass, A.; Bürgi, T. First Enantioseparation and Circular Dichroism Spectra of Au38 Clusters Protected by Achiral Ligands. Nat. Commun. 2012, 3, 798.

(9) Knoppe, S.; Dolamic, I.; Bürgi, T. Racemization of a Chiral Nanoparticle Evidences the Flexibility of the Gold-Thiolate Interface. J. Am. Chem. Soc. 2012, 134, 13114-13120.

(10) Malola, S.; Häkkinen, H. Chiral Inversion of Thiolate-Protected Gold Nanoclusters via Core Reconstruction Without Breaking a $\mathrm{Au}-$ S Bond. J. Am. Chem. Soc. 2019, 141, 6006-6012.

(11) Dolamic, I.; Varnholt, B.; Bürgi, T. Chirality Transfer from Gold Nanocluster to Adsorbate Evidenced by Vibrational Circular Dichroism. Nat. Commun. 2015, 6, 7117.

(12) Sadlej, J.; Dobrowolski, J. C.; Rode, J. E. VCD Spectroscopy as a Novel Probe for Chirality Transfer in Molecular Interactions. Chem. Soc. Rev. 2010, 39, 1478-1488.

(13) Mujica, V. Nano-Plasmonics: Chirality Transfer Takes a Jump. Nat. Chem. 2015, 7, 543-544.

(14) Qian, H.; Zhu, M.; Gayathri, C.; Gil, R. R.; Jin, R. Chirality in Gold Nanoclusters Probed by NMR Spectroscopy. ACS Nano 2011, 5, 8935-8942.

(15) Salassa, G.; Bürgi, T. NMR Spectroscopy: A Potent Tool for Studying Monolayer-Protected Metal Nanoclusters. Nanoscale Horizons 2018, 2, 457-463.

(16) Salorinne, K.; Lahtinen, T.; Malola, S.; Koivisto, J.; Häkkinen, H. Solvation Chemistry of Water-Soluble Thiol-Protected Gold Nanocluster $\mathrm{Au}_{102}$ from DOSY NMR Spectroscopy and DFT Calculations. Nanoscale 2014, 6, 7823-7826.

(17) Salorinne, K.; Malola, S.; Wong, O. A.; Rithner, C. D.; Chen, X.; Ackerson, C. J.; Häkkinen, H. Conformation and Dynamics of the Ligand Shell of a Water-Soluble $\mathrm{Au}_{102}$ Nanoparticle. Nat. Commun. 2016, 7, 10401.

(18) Dainese, T.; Antonello, S.; Bogialli, S.; Fei, W.; Venzo, A.; Maran, F. Gold Fusion: From $\mathrm{Au}_{25}(\mathrm{SR})_{18}$ to $\mathrm{Au}_{38}(\mathrm{SR})_{24}$, the Most Unexpected Transformation of a Very Stable Nanocluster. ACS Nano 2018, 12, 7057-7066.

(19) Riccardi, L.; Gabrielli, L.; Sun, X.; De Biasi, F.; Rastrelli, F.; Mancin, F.; De Vivo, M. Nanoparticle-Based Receptors Mimic Protein-Ligand Recognition. Chem. 2017, 3, 92-109.

(20) Clore, G. M.; Szabo, A.; Bax, A.; Kay, L. E.; Driscoll, P. C.; Gronenborn, A. M. Deviations from the Simple Two-Parameter Model-Free Approach to the Interpretation of Nitrogen-15 Nuclear Magnetic Relaxation of Proteins. J. Am. Chem. Soc. 1990, 112, 49894991.

(21) Lipari, G.; Szabo, A. Model-Free Approach to the Interpretation of Nuclear Magnetic Resonance Relaxation in Macromolecules. 1. Theory and Range of Validity. J. Am. Chem. Soc. 1982, 104, 4546-4559.

(22) Pohjolainen, E.; Chen, X.; Malola, S.; Groenhof, G.; Häkkinen, H. A Unified AMBER-Compatible Molecular Mechanics Force Field for Thiolate-Protected Gold Nanoclusters. J. Chem. Theory Comput. 2016, 12, 1342-1350.

(23) Jorgensen, W. L. Pressure Dependence of the Structure and Properties of Liquid N-Butane. J. Am. Chem. Soc. 1981, 103 (16), 4721-4726.

(24) Bonham, R. A.; Bartell, L. S. The Molecular Structure and Rotational Isomerization of N-Butane 1,2. J. Am. Chem. Soc. 1959, 81, 3491-3496.

(25) De Nardi, M.; Antonello, S.; Jiang, D. E.; Pan, F.; Rissanen, K.; Ruzzi, M.; Venzo, A.; Zoleo, A.; Maran, F. Gold Nanowired : A Linear $\left(\mathrm{Au}_{25}\right)_{\mathrm{n}}$ Polymer from $\mathrm{Au}_{25}$ Molecular Clusters. ACS Nano 2014, 8, $8505-8512$.

(26) Knoppe, S.; Dharmaratne, A. C.; Schreiner, E.; Dass, A.; Bürgi, T. Ligand Exchange Reactions on $\mathrm{Au}(38)$ and $\mathrm{Au}(40)$ Clusters: $\mathrm{A}$ 
Combined Circular Dichroism and Mass Spectrometry Study. J. Am. Chem. Soc. 2010, 132, 16783-16789.

(27) Stellwagen, D.; Weber, A.; Bovenkamp, G. L.; Jin, R.; Bitter, J. H.; Kumar, C. S. S. R. Ligand Control in Thiol Stabilized Au38 Clusters. RSC Adv. 2012, 2, 2276-2283.

(28) Knoppe, S.; Boudon, J.; Dolamic, I.; Dass, A.; Bürgi, T. Size Exclusion Chromatography for Semipreparative Scale Separation of $\mathrm{Au}_{38}(\mathrm{SR})_{24}$ and $\mathrm{Au}_{40}(\mathrm{SR})_{24}$ and Larger Clusters. Anal. Chem. 2011, 83, 5056-5061.

(29) Robinson, P. T.; Pham, T. N.; Uhrín, D. In Phase Selective Excitation of Overlapping Multiplets by Gradient-Enhanced Chemical Shift Selective Filters. J. Magn. Reson. 2004, 170, 97-103.

(30) Hall, L. D.; Norwood, T. J. An Improved Chemical-ShiftSelective Filter. J. Magn. Reson. 1988, 78, 582-587.

(31) Duncan, S. J.; Lewis, R.; Bernstein, M. A.; Sandor, P. Selective Excitation of Overlapping Multiplets; the Application of Doubly Selective and Chemical Shift Filter Experiments to Complex NMR Spectra. Magn. Reson. Chem. 2007, 45, 283-288.

(32) Dickson, C. J.; Madej, B. D.; Skjevik, Å. A.; Betz, R. M.; Teigen, K.; Gould, I. R.; Walker, R. C. Lipid14: The Amber Lipid Force Field. J. Chem. Theory Comput. 2014, 10, 865-879.

(33) Bayly, C. I.; Cieplak, P.; Cornell, W.; Kollman, P. A. A WellBehaved Electrostatic Potential Based Method Using Charge Restraints for Deriving Atomic Charges: The RESP Model. J. Phys. Chem. 1993, 97, 10269-10280.

(34) Vanquelef, E.; Simon, S.; Marquant, G.; Garcia, E.; Klimerak, G.; Delepine, J. C.; Cieplak, P.; Dupradeau, F.-Y. R.E.D. Server: A Web Service for Deriving RESP and ESP Charges and Building Force Field Libraries for New Molecules and Molecular Fragments. Nucleic Acids Res. 2011, 39, W511-W517 accessed May 17, 2016.

(35) Hess, B.; Kutzner, C.; van der Spoel, D.; Lindahl, E. GROMACS 4: Algorithms for Highly Efficient, Load-Balanced, and Scalable Molecular Simulation. J. Chem. Theory Comput. 2008, 4, 435-447. 